\title{
Dynamic SpoIIIE assembly mediates septal membrane fission during Bacillus subtilis sporulation
}

\author{
Tinya C. Fleming, ${ }^{1}$ Jae Yen Shin, ${ }^{2,3}$ Sang-Hyuk Lee, ${ }^{2,4}$ Eric Becker, ${ }^{1}$ Kerwyn Casey Huang, $^{5}$ \\ Carlos Bustamante, ${ }^{2,3,4,6,7}$ and Kit Pogliano ${ }^{1,8}$ \\ ${ }^{1}$ Division of Biological Sciences, University of California at San Diego, La Jolla, California 92093, USA; ${ }^{2}$ Department of Physics, \\ University of California at Berkeley, Berkeley, California 94720, USA; ${ }^{3}$ Howard Hughes Medical Institute, University of \\ California at Berkeley, Berkeley, California 94720, USA; ${ }^{4}$ Physical Bioscience Division, Lawrence Berkeley National Laboratory, \\ Berkeley, California 94720, USA; ${ }^{5}$ Department of Bioengineering, Stanford University, Stanford, California 94305, USA; \\ ${ }^{6}$ Department of Molecular and Cell Biology, University of California at Berkeley, Berkeley, California 94720, USA; \\ ${ }^{7}$ Department of Chemistry, University of California at Berkeley, Berkeley, California 94720, USA
}

SpoIIIE is an FtsK-related protein that transports the forespore chromosome across the Bacillus subtilis sporulation septum. We use membrane photobleaching and protoplast assays to demonstrate that SpoIIIE is required for septal membrane fission in the presence of trapped DNA, and that DNA is transported across separate daughter cell membranes, suggesting that SpoIIIE forms a channel that partitions the daughter cell membranes. Our results reveal a close correlation between septal membrane fission and the assembly of a stable SpoIIIE translocation complex at the septal midpoint. Time-lapse epifluorescence, total internal reflection fluorescence (TIRF) microscopy, and live-cell photoactivation localization microscopy (PALM) demonstrate that the SpoIIIE transmembrane domain mediates dynamic localization to active division sites, whereas the assembly of a stable focus also requires the cytoplasmic domain. The transmembrane domain fails to completely separate the membrane, and it assembles unstable foci. TIRF microscopy and biophysical modeling of fluorescence recovery after photobleaching (FRAP) data suggest that this unstable protein transitions between disassembled and assembled oligomeric states. We propose a new model for the role of SpoIIIE assembly in septal membrane fission that has strong implications for how the chromosome terminus crosses the septum.

[Keywords: SpoIIIE; DNA translocase; membrane fission; FRAP; PALM]

Supplemental material is available at http://www.genesdev.org.

Received March 12, 2010; revised version accepted April 15, 2010.

The final step of cell division is the separation of daughter cell membranes (septal membrane fission). Although septal membrane fission is universally conserved in all cellular organisms, it remains poorly understood, particularly in prokaryotes. Septal membrane fission in plant and animal cells requires vesicular transport, and it is likely that fusion of vesicles that span the intracellular bridge completes cell division (Finger and White 2002; Jurgens 2005). Bacterial division commences with membrane constriction, as in animal cells, but there is no evidence for vesicular transport in bacteria, suggesting that they mediate septal membrane fission by a fundamentally different mechanism than eukaryotes.

Bacillus subtilis sporulation provides a unique opportunity to study two distinct membrane fission events in

${ }^{8}$ Corresponding author.

E-MAIL kpogliano@ucsd.edu; FAX (858) 822-5740.

Article is online at http://www.genesdev.org/cgi/doi/10.1101/gad.1925210. a bacterium. First, at the onset of sporulation, the cell divides near the cell pole and traps one chromosome in the septum (Fig. 1A). Septal membrane fission is therefore complicated by this trapped chromosome, which is translocated into the forespore by the SpoIIIE DNA translocase (Wu and Errington 1994; Errington 2001). Next, the membrane of the larger mother cell migrates around the smaller forespore in a phagocytosis-like process called engulfment (for review, see Errington 2003; Hilbert and Piggot 2004). Ultimately the leading edges of the mother cell membrane converge on the distal side of the forespore, and a second membrane fission event pinches off a detached, internalized forespore (Sharp and Pogliano 1999).

Prior data suggested that both membrane fission events of sporulation involve the SpoIIIE DNA translocase. SpoIIIE is required for cytoplasmic compartmentalization of the two daughter cells (Wu and Errington 1994; Liu et al. 2006) and for engulfment membrane fission (Sharp 

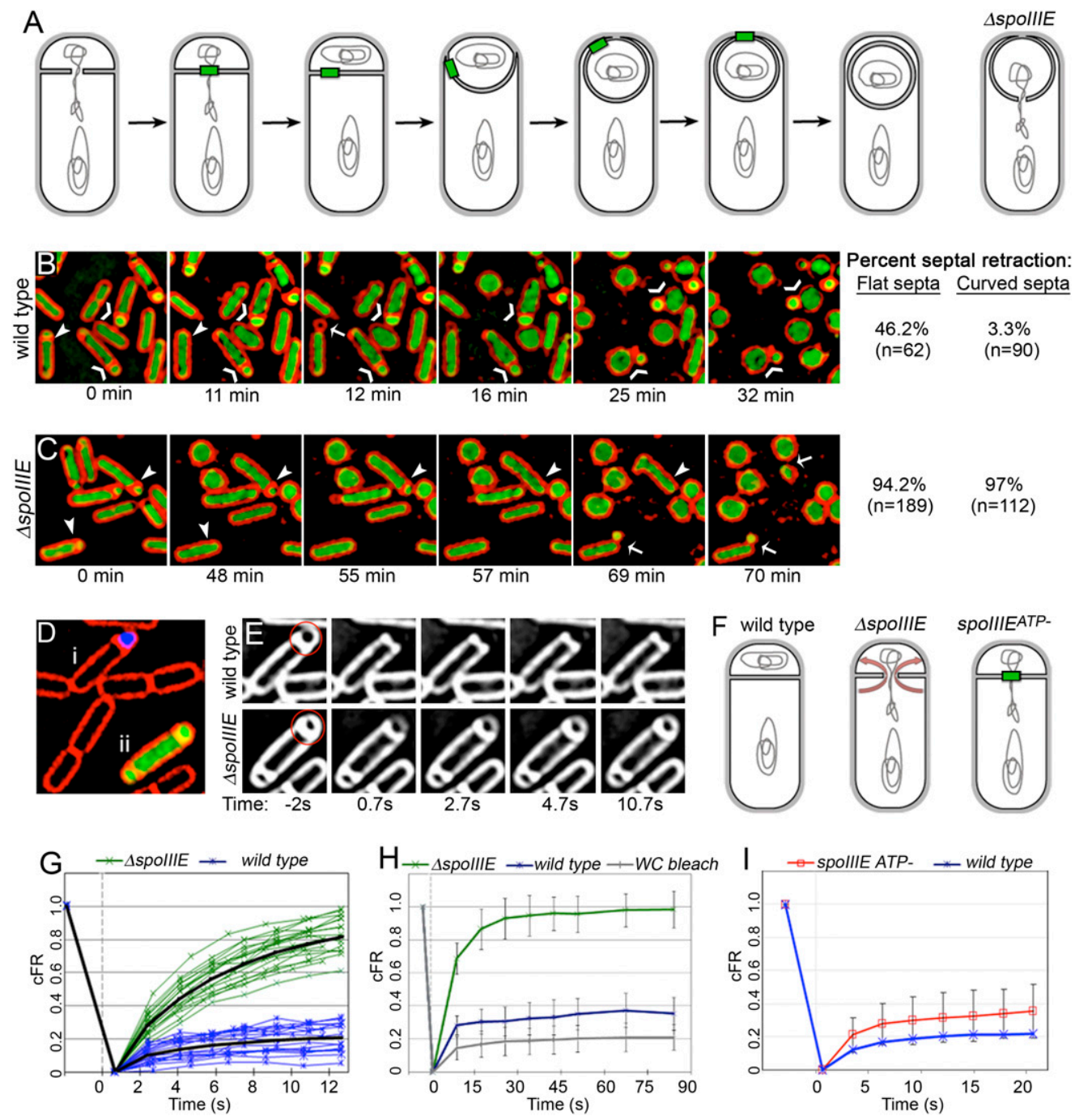

Figure 1. SpoIIIE is required for septal membrane fission. (A) Cartoon showing membrane and DNA dynamics during sporulation and the structure of $\Delta$ spoIIIE sporangia. SpoIIIE is shown in green (Wu and Errington 1997; Sharp and Pogliano 1999). (B,C) Protoplast assays for septal membrane fission in wild type $(B)$ and $\Delta$ spoIIIE $(C)$, showing cell membranes (red) and DNA (green). (Chevrons) Sporangia that generated separate protoplasts; (arrowheads) septa that retracted; (arrows) protoplasts that separated after septal retraction (see also Supplemental Movies S1, S2). (D,E) FRAP assays for septal membrane fission on a mixed culture of CFP-tagged wild-type (blue) and YFP-tagged $\Delta$ spoIIIE (green) cells. (D) Prebleach image also showing FM 4-64-stained membranes in red. (E) Membranes (white) imaged at various times show no recovery for wild type (top) and rapid recovery for $\Delta$ spoIIIE (bottom) forespore membrane fluorescence. Photobleaching of the bleached region (red circle) occurred at 0 sec. $(F)$ Diagrams illustrate membrane structure of wild type and $\Delta$ spoIIIE allowing diffusion from the mother cell to the forespore (arrows), and SpoIIIE ${ }^{\text {ATP- }}$ (green) blocking diffusion. (G) Plot showing the cFR (see the Materials and Methods) of forespore membrane fluorescence for wild-type (blue) and $\Delta$ spoIIIE (green) sporangia. Each green and blue line represents fluorescence recovery of an individual forespore. The thick black lines show the average recovery for each genotype. $(H)$ Plot showing the average cFR and standard deviation of wild-type, $\Delta$ spoIIIE, and whole-cell bleach events (WC bleach) for $84 \mathrm{sec}$ of recovery time. (I) Average cFR for $\operatorname{spoIIIE}^{A T P-}$ (red) with standard deviation error bars compared with wild-type average (blue).

and Pogliano 1999, 2003; Liu et al. 2006), suggesting that SpoIIIE might play a general role in membrane fission during sporulation. The localization of SpoIIIE to both the septal midpoint (the site of DNA translocation) and to the pole of the engulfed forespore before engulfment membrane fission indicates that the protein is in the right place at the right time to directly participate in both events (Sharp and Pogliano 1999; Wu and Errington 1997).
In addition, genetic studies have demonstrated that DNA translocation depends primarily on the cytoplasmic motor domain, while compartmentalization and engulfment membrane fission depend primarily on the $\mathrm{N}$-terminal transmembrane domain (Sharp and Pogliano 1999, 2003; Liu et al. 2006), indicating that these two functions are genetically separable. The close correlation between defects in cytoplasmic compartmentalization 
and in engulfment membrane fission suggested that the cytoplasmic compartmentalization defect of spoIIIE mutants might be due to incomplete septal membrane fission (Liu et al. 2006).

Based on these results, we proposed that, when the sporulation septum completes constriction, SpoIIIE assembles a transmembrane channel that insulates the negatively charged DNA from the hydrophobic lipid bilayer and its hydrophilic head groups. One model for this structure is a paired transmembrane channel with subunits in both daughter cell membranes encircling the trapped DNA strands (Liu et al. 2006), which could also mediate the temporary partitioning of the forespore and mother cell membrane during DNA translocation. In support of this model, a recent study found that wild-type sporangia displayed compartmentalized forespore membranes during DNA translocation, and that a translocasedefective, localization-proficient spoIIIE mutant maintained partitioned membranes when DNA traversed the septum (Burton et al. 2007). However, this study also concluded that SpoIIIE was not required for septal membrane fission, based on observations suggesting a $\Delta$ spoIIIE mutant showed normal septal membrane fission (Burton et al. 2007).

Here we confirm the observation that SpoIIIE maintains membrane compartmentalization during DNA translocation. However, protoplast and fluorescence recovery after photobleaching (FRAP) assays demonstrate that, in the absence of SpoIIIE, the forespore and mother cell membranes remain contiguous, indicating defects in septal membrane fission. Biophysical modeling of lipid diffusion indicates that FRAP can be used as a quantitative assay for membrane geometry and compartmentalization. In addition, we compare FRAP results with assessments of SpoIIIE assembly by several microscopy techniques and find a correlation between the ability of SpoIIIE to assemble and its ability to partition daughter cell membranes. Our data indicate that SpoIIIE assembly is a multistep process, with initial dynamic localization to sites of active cell division mediated by the transmembrane domain, followed by the assembly of a stable translocation complex, which requires both the transmembrane and the cytoplasmic motor domain. These results resolve conflicting observations regarding the role of the transmembrane and motor domains in SpoIIIE assembly, and provide mechanistic insight into septal membrane fission during sporulation.

\section{Results}

SpoIIIE is required for septal membrane fission during sporulation

We used two methods to determine if SpoIIIE is required for septal membrane fission. First, we treated cells with lysozyme to digest peptidoglycan, which causes rodshaped Bacillus cells to become spherical protoplasts. If septal membrane fission is complete, the forespore will form a separate protoplast from the mother cell. If septal membrane fission is incomplete, the septum will retract as peptidoglycan is digested. This protoplast assay showed that $46 \%$ of flat septa in wild-type cells retract (Fig. 1B, arrowhead), suggesting that many had incomplete septa with septal openings smaller than the resolution limit of epifluorescence microscopy. Engulfment starts after septation, and is readily visualized by the curving of the septum around the forespore. We found that just $3 \%$ of curved wild-type septa retracted, confirming that septation was incomplete in very few cells. In contrast, $97 \%$ of curved septa in $\Delta$ spoIIIE cells retracted (Fig. 1C, arrowheads). Thus, even after the onset of engulfment, most $\Delta$ spoIIIE sporangia fail to complete septal membrane fission.

Protoplast assays also appeared to subject cells to physical stresses that can pinch off several separated protoplasts from a single cell. Specifically, some sporangia first show septal retraction and later pinch off a foresporesized protoplast at the forespore pole (Fig. 1B,C, arrows). We therefore used a second, more direct method: staining cells with the membrane dye FM 4-64, and then performing a FRAP analysis by photobleaching only the forespore membrane (Fig. 1D,E). If septal membrane fission is complete, then the forespore membrane fluorescence will fail to recover because the dye will be unable to diffuse across the septum. However, if septal membrane fission is incomplete, forespore and mother cell membranes remain connected, allowing FM 4-64 to diffuse from the unbleached mother cell into the bleached forespore (Fig. $1 \mathrm{~F})$. In this case, forespore fluorescence should fully recover due to equilibration of membrane dye between the forespore and mother cell.

We performed simultaneous FRAP of forespore membranes of wild-type sporangia expressing a CFP reporter and $\Delta$ spoIIIE sporangia expressing a YFP reporter to observe fluorescence recovery side by side (Fig. 1D). We bleached forespores with slightly curved septa to select sporangia that had completed cell division. Rapid recovery of forespore fluorescence was observed in only the $\Delta$ spoIIIE strain, suggesting that the membranes of this strain are contiguous, whereas wild-type membranes are separated (Fig. 1E). Identical results were obtained with two published spoIIIE deletions (Wu and Errington 1994; Pogliano et al. 1997; Sharp and Pogliano 1999) and with the complete deletion of spoIIIE constructed for this study (Supplemental Figure S1).

We next quantified the results in a manner that allows the results of many individual cells to be overlayed and the average recovery and standard deviations to be calculated (Fig. 1G-I). FRAP analysis of wild type showed limited recovery, with an average recovery of $20 \%$ membrane fluorescence (corrected fraction recovery $[\mathrm{cFR}]=$ 0.2 ) after $12 \mathrm{sec}$ (Fig. 1G) and 35\% after $84 \mathrm{sec}$ (Fig. 1H). The whole-cell bleach control also showed a slight recovery (cFR $=0.2$ at $84 \mathrm{sec})$, perhaps due to exchange of fluorescent FM 4-64 from the medium to the cells or due to reversible photobleaching of some FM 4-64 molecules. The slightly greater fluorescence recovery of bleached wild-type forespores than bleached whole cells likely results from incomplete septal bleaching, since the forespore membrane cannot be distinguished from the 
mother cell membrane at the septum. The failure of wildtype forespores to fully recover indicates that the forespore and mother cell membranes are separated (Fig. 1F). In contrast, analysis of $\Delta$ spoIIIE sporangia showed complete recovery to $100 \%$ of possible fluorescence $(\mathrm{cFR}=1$ ) with an average time to half recovery $\left(\tau_{1 / 2}\right)$ of $\sim 5 \mathrm{sec}$ (Fig. $1 \mathrm{G}-\mathrm{H})$. This indicates that the forespore and mother cell membranes remain contiguous in the absence of SpoIIIE (Fig. 1F). Thus, two separate assays demonstrate that SpoIIIE is required for septal membrane fission during $B$. subtilis sporulation.

SpoIIIE is required for septal membrane fission only in the presence of trapped DNA

We next tested whether SpoIIIE was required for septal membrane fission in the absence of trapped DNA. To do so, we used a racA-null mutation, which causes defects in polar chromosome anchoring during sporulation and produces $\sim 50 \%$ anucleate forespores (Ben-Yehuda et al. 2003b; Wu and Errington 2003). We performed protoplast and FRAP experiments on $\triangle \mathrm{rac} A$ and $\Delta \mathrm{rac} A \Delta$ spoIIIE cells stained with FM 4-64 and the DNA stain Sytox Green (Becker and Pogliano 2007) to discriminate between sporangia with or without DNA in the forespore. FRAP analysis of racA sporangia showed no significant recovery of forespore fluorescence, independent of DNA trapped in the forespore (Fig. 2A). Thus, the racA deletion does not affect septal fission. Two outcomes were observed with $\Delta$ racA $\Delta$ spoIIIE forespores (Fig. 2B,C). First, sporangia with DNA in the forespore showed a rapid fluorescence recovery similar to $\Delta$ spoIIIE, consistent with a requirement for SpoIIIE in septal fission when DNA traverses the septum. Second, sporangia with no detectable DNA in the forespore showed incomplete recovery similar to wild type, indicating that septal membrane fission was complete in the absence of trapped DNA (Fig. 2B,C). Similar results were obtained with the protoplast assay (Supplemental Fig. S2; Supplemental Movies S3, S4). Thus, SpoIIIE is required for septal membrane fission only when DNA traverses the septum, suggesting that there is another mechanism for septal membrane fission in the absence of trapped DNA.
SpoIIIE partitions the membrane while DNA traverses the septum

To determine if SpoIIIE partitions daughter cell membranes while DNA crosses the septum or after DNA translocation is complete, we blocked DNA translocation with the G467S single-base-pair mutation in a conserved region of the spoIIIE ATPase motor domain. The SpoIIIE ${ }^{\mathrm{ATP}-}$ protein can still assemble at the septum and mediate cytoplasmic compartmentalization, but cannot translocate DNA, and the forespore chromosome remains trapped in the septum (Wu and Errington 1994; Sharp and Pogliano 1999). spoIIIE ${ }^{A T P-}$ showed a slow and limited fluorescence recovery in FRAP similar to wild type (Fig. 1I). This suggests that assembly of SpoIIIE ${ }^{\text {ATP- }}$ around the DNA provides a barrier to FM 4-64 diffusion between the forespore and mother cell membranes even when SpoIIIE is unable to translocate DNA. This finding is consistent with previous results (Burton et al. 2007), and indicates that the SpoIIIE DNA translocation complex can partition daughter cell membranes.

\section{Role of the SpoIIIE transmembrane domain in membrane fission}

We next investigated the role of the SpoIIIE transmembrane domain (SpoIIIE ${ }^{1-192}$ ) in septal membrane fission by performing FRAP analyses on cells expressing various spoIIIE transmembrane mutants. Previous data indicated that SpoIIIE ${ }^{1-192}$ localizes to the septum, supports cytoplasmic compartmentalization in $95 \%$ of sporangia, and partially rescues engulfment membrane fission from $1 \%$ in $\Delta$ spoIIIE to $13 \%$ (Bath et al. 2000; Sharp and Pogliano 2003). We therefore predicted that expressing only the transmembrane domain would at least partially rescue the septal membrane fission defect of the null strain. FRAP analysis of spoIIIE ${ }^{1-192}$ sporangia produced a broad range of cFR curves ranging from no recovery, similar to wild-type cells, to full recovery, similar to $\Delta$ spoIIIE cells (Fig. 3A). Additional FRAP experiments demonstrated that cells with intermediate recoveries continued to recover for at least $180 \mathrm{sec}$ (data not shown). This variable outcome (which is addressed below) indicates that SpoIIIE ${ }^{1-192}$ only partially supports septal membrane
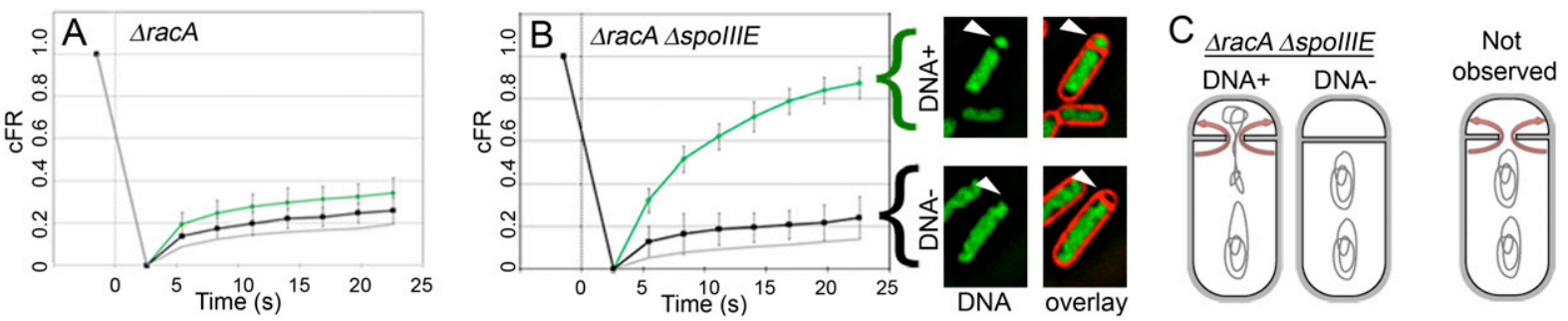

Figure 2. Septal fission defects occur only in $\Delta$ spoIIIE sporangia with trapped DNA. (A) Plot of cFR for $\Delta$ racA sporangia with (green) or without (black) DNA in the forespore show similarly limited fluorescence recovery. (Gray curve) Whole-cell bleach control. (B) Plot of cFR for $\Delta$ spoIIIE $\triangle \mathrm{racA}$ sporangia with DNA in the forespore (green) show recovery, and those without DNA (black) show limited recovery similar to wild type. (C) Cartoon showing possible membrane and DNA configurations for $\Delta$ spoIIIE $\Delta$ racA and the impact on FM 4-64 diffusion (arrows). 

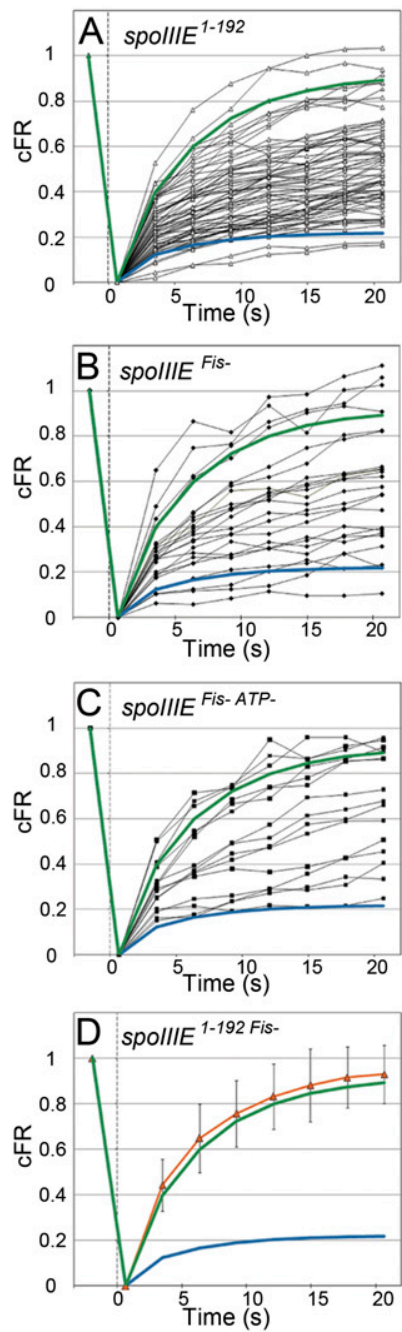
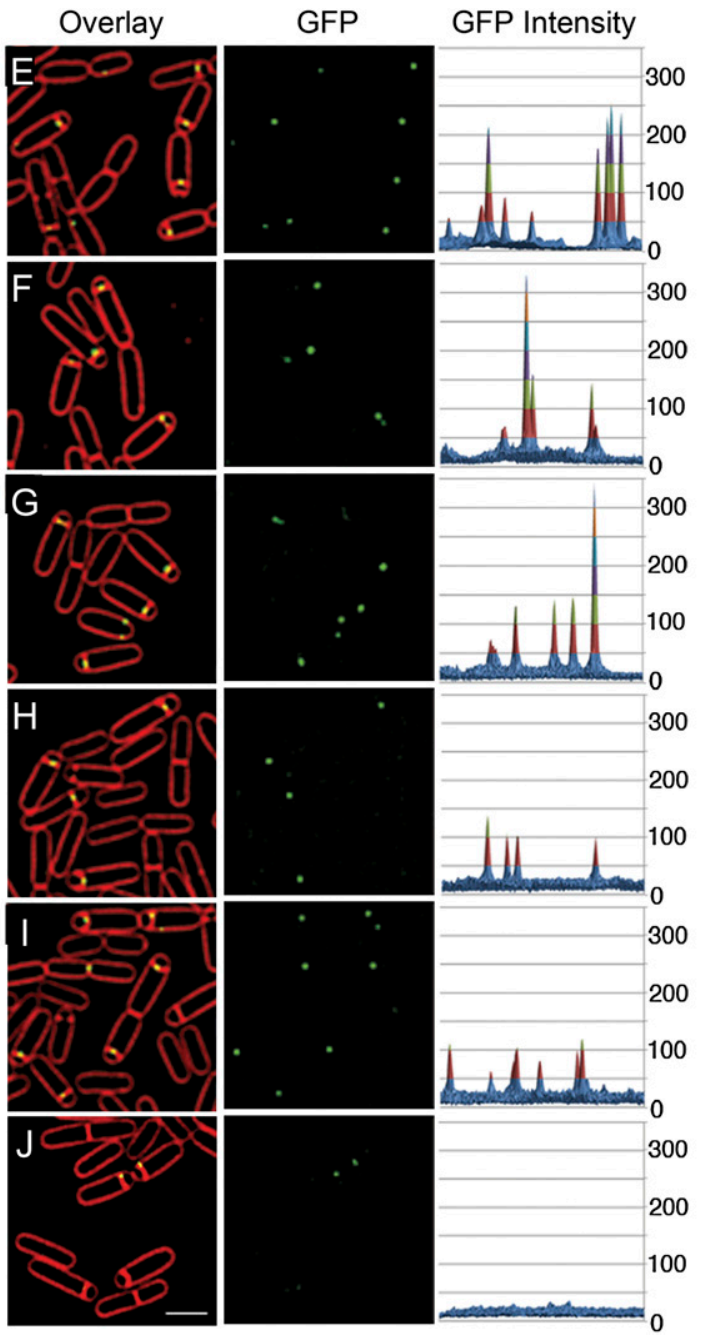
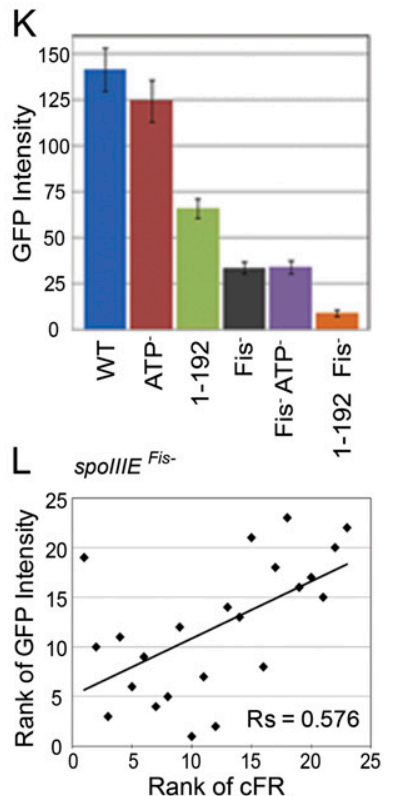

M spollIE Fis-ATP-

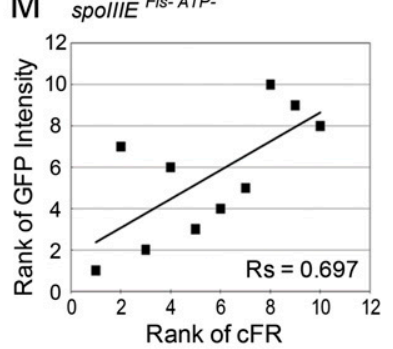

Figure 3. Role of transmembrane and cytoplasmic domains in septal fission and SpoIIIE assembly. $(A-D)$ Average cFR for wild type (blue) and $\Delta$ spoIIIE (green) are shown as references. Plot of cFR for individual cells (black lines) expressing $\operatorname{SpoIIIE}^{1-192}(A)$, SpoIIIE ${ }^{\text {Fis- }}$ $(B)$, and SpoIIIE Fis-ATP- $_{(C)}$. (D) Average cFR and standard deviations for spoIIIE ${ }^{1-192}$ Fis- (orange). (E-J) Fluorescent images of membrane $\left(\right.$ red) and SpoIIIE-GFP (green) for wild type $(E)$, SpoIIIE ${ }^{\mathrm{ATP}-}(F)$, $\operatorname{SpoIIIE}^{1-192}(G)$, $\operatorname{SpoIIIE}^{\mathrm{Fis}-}(H)$, SpoIIIE ${ }^{\mathrm{Fis}-\mathrm{ATP}-}(I)$, and SpoIIIE ${ }^{1-192}$ Fis- $(J)$. The third panel is a three-dimensional plot of the normalized GFP intensity in each field. $(K)$ Histogram of average GFP intensity for wild-type and mutant proteins, with $99 \%$ CI error bars. $(L, M)$ Rank correlation plot of GFP focus intensity versus FRAP recovery in individual cells expressing SpoIIIE ${ }^{\mathrm{Fis}-}-\mathrm{GFP}(L)$ and SpoIIIE ${ }^{\mathrm{Fis}-\mathrm{ATP}-}$ GFP $(M)$, with the brightest focus and the lowest cFR ranked separately as 1 (Supplemental Table S1). The black line of best fit and Spearman's rank correlation (Rs values of 0.576 and 0.697 , with $P<0.05$ for SpoIIIE ${ }^{\mathrm{Fis}}$ and SpoIIIE ${ }^{\mathrm{Fis}-\mathrm{ATP}}$ ) show a correlation between dim foci and rapid recovery.

fission, and that the cytoplasmic domain is also involved in septal membrane fission.

We further investigated the role of the transmembrane domain in septal membrane fission by performing FRAP experiments on a previously isolated epitope insertion in the SpoIIIE transmembrane domain (spoIIIE124::i31, here referred to as spoIIIE $E^{F i s-}$ ) that partially blocks engulfment membrane fission and cytoplasmic compartmentalization without preventing DNA translocation (Liu et al. 2006). FRAP analysis of spoIIIE ${ }^{\mathrm{Fis}-}$ sporangia also showed a broad range of cFR curves, from little or no recovery to complete recovery (Fig. 3B), providing further support for the importance of the transmembrane domain in membrane fission.

The effects of the spoIIIE ${ }^{\text {Fis- }}$ mutation on septal membrane fission could occur either after the completion of DNA translocation, as proposed previously by Liu et al. (2006), or during DNA translocation. To discriminate between these two hypotheses, we combined the spoIIIE(G467S) mutation that blocks DNA translocation to trap DNA across the septum with the SpoIIIE124::i31 mutation to generate a spoIIIE ${ }^{\text {Fis-ATP- }}$ strain. Performing FRAP on spoIIIE ${ }^{\mathrm{Fis}-\mathrm{ATP}-}$ sporangia produced the same wide range of cFR curves as in the single SpoIIIE ${ }^{\mathrm{Fis}-}$ mutant (Fig. 3C), indicating that SpoIIIE ${ }^{\text {Fis-ATP- }}$ fails to support membrane fission while DNA crosses the septum. Thus, the spoIIIE ${ }^{\mathrm{Fis}-}$ mutation affects the ability of the protein to mediate septal fission before or during DNA translocation, rather than afterward.

The observation that the SpoIIIE transmembrane domain (SpoIIIE $\left.{ }^{1-192}\right)$ did not fully support membrane 
fission suggests that the cytoplasmic domain contributes to septal membrane fission. To further investigate the respective contributions of the cytoplasmic and transmembrane domains to SpoIIIE assembly, we deleted the cytoplasmic domain from SpoIIIE ${ }^{\mathrm{Fis}-}$ to produce SpoIIIE ${ }^{1-192}$ Fis- $^{-}$. FRAP analysis of this strain demonstrated rapid recovery similar to the spoIIIE-null strain (Fig. 3D), with no sporangia showing an intermediate recovery as in the full-length SpoIIIE ${ }^{\text {Fis- }}$ protein or SpoIIIE ${ }^{1-192}$. Thus, deletion of the cytoplasmic domain from a partially fission-defective mutant enhances the membrane fission defect. These results suggest that the transmembrane domain and the cytoplasmic domain cooperate to mediate assembly of a SpoIIIE complex that can partition the forespore and mother cell membranes.

\section{Correlation of mutant SpoIIIE protein assembly and FRAP recovery}

The wide range of FRAP recovery curves suggested that individual cells might vary in their ability to assemble a SpoIIIE DNA translocation complex that mediates septal membrane fission. To test this hypothesis, we performed two experiments to determine if there was a correlation between the ability of various SpoIIIE mutant proteins to assemble at the septum and their ability to complete septal membrane fission. First, we inferred the state of SpoIIIE assembly at the septal midpoint by quantifying the fluorescence intensity of wild-type and mutant SpoIIIEGFP foci. The two membrane fission-proficient proteins, wild-type SpoIIIE-GFP and SpoIIIE ${ }^{\mathrm{ATP}-}$-GFP, showed equivalently high GFP focus intensities (Fig. 3E,F,K). Two mutations that cause intermediate septal fission defects, spoIIIE $^{1-192}$ and spoIIIE ${ }^{\text {Fis- }}$, caused a decrease in average fluorescence intensities to $53 \%$ and $24 \%$ of wild-type levels, respectively (Fig. 3G-I,K). The mutant completely defective for membrane fission, SpoIIIE ${ }^{1-192}$ Fis-GFP, assembled few detectable foci, which were very dim (Fig. $3 \mathrm{~J}, \mathrm{~K})$. These results suggest a correlation between decreasing GFP focus intensity and a decreased ability to mediate membrane fission, which we verified does not result from changes in proteins levels (Supplemental Fig. S3). Second, we assessed the correlation between GFP focus intensity and FRAP outcomes in individual cells of the spoIIIE ${ }^{\text {Fis- }}$ and spoIIIE $E^{\text {Fis-ATP- }}$ strains, which show a broad range of FRAP recovery curves and focus intensities. A Spearman's rank correlation test indicated a significant correlation between the assembly of a bright SpoIIIE focus at the septum and membrane compartmentalization (Fig. 3L,M) in which cells with dimmer GFP foci tended to have faster FRAP recovery rates (Supplemental Table S1). The correlation between GFP focus intensity and FRAP recovery suggests a direct involvement of SpoIIIE in septal membrane fission during sporulation.

\section{Multistep pathway for SpoIIIE assembly}

To further explore the connection between SpoIIIE assembly and membrane fission, we used fluorescence microscopy to follow SpoIIIE assembly. We first performed time-lapse epifluorescence microscopy over a comparatively long time scale of at least $30 \mathrm{~min}$. This revealed a dynamic population of SpoIIIE molecules at the division site both before and during constriction (Fig. 4A; Supplemental Fig. S4). The SpoIIIE focus was first observed $\sim 3$ min after the apparent completion of septation (Fig. 4A), although this estimate is imprecise due to the limited spatial resolution of epifluorescence microscopy, which limits the ability to visualize small openings in the septum. Nevertheless, this suggests that the SpoIIIE focus rapidly assembles from a dynamic population of molecules at the septum.

We next used total internal reflection fluorescence (TIRF) microscopy to explore SpoIIIE dynamics with increased temporal resolution. The increased sensitivity of TIRF allows visualization of fewer molecules, and a shorter exposure time allows visualization of rapidly moving molecules. To compensate for the shallow illumination plane, we used partial TIRF in which the laser was aligned so that stable SpoIIIE foci at the septal midpoint ( $\sim 00 \mathrm{~nm}$ into the cell) were clearly imaged. TIRF imaging demonstrated that SpoIIIE initially localizes to incomplete septa as dynamic foci (Fig. 4B,D). Septal kymographs, in which slices across the septum at each time point are cropped and stacked, more clearly demonstrated movement of SpoIIIE, and suggested that foci either assembled and disassembled on opposite sides of the septum, or moved from one side to the other within seconds (Fig. 4B,D). Thus, the first step of SpoIIIE assembly is dynamic localization to active division sites, which is challenging to visualize with epifluorescence microscopy but is clearly imaged by TIRF. Cells with flat septa showed two distinct patterns. Most (85\%) showed a stable focus that moved little throughout the experiment (Fig. 4C,E). However, $\sim 15 \%$ showed SpoIIIE-GFP that moved within the septum (Fig. 4E), indicating that either septation is not fully complete in these cells, or the SpoIIIE focus has not yet fully assembled, as confirmed by live-cell photoactivation localization microscopy (PALM) (Fig. 5).

\section{The transmembrane domain assembles an unstable focus}

We next examined the dynamics of the SpoIIIE transmembrane domain by TIRF microscopy. SpoIII ${ }^{1-192}$-GFP molecules showed dynamic localization to incomplete septa that was indistinguishable from the wild type (Fig. 4F), indicating that the transmembrane domain is sufficient for dynamic septal localization. Cells in which septation appeared to be complete showed dynamic assemblies and foci in frequencies similar to wild type. However, septal kymographs revealed that SpoIIIE ${ }^{1-192}$-GFP foci were less stable than the wild-type foci. Specifically, SpoIIIE ${ }^{1-192}$-GFP foci often showed frequent flares emanating out from the central focus (Fig. 4G). Indeed, even the most stable transmembrane domain focus observed (Fig. 4G, cell 6) showed an occasional diffuse focus not seen in wild type. This suggests that the SpoIIE transmembrane domain assembles unstable foci. 
Fleming et al.

\section{A Timelapse epifluorescence microscopy}

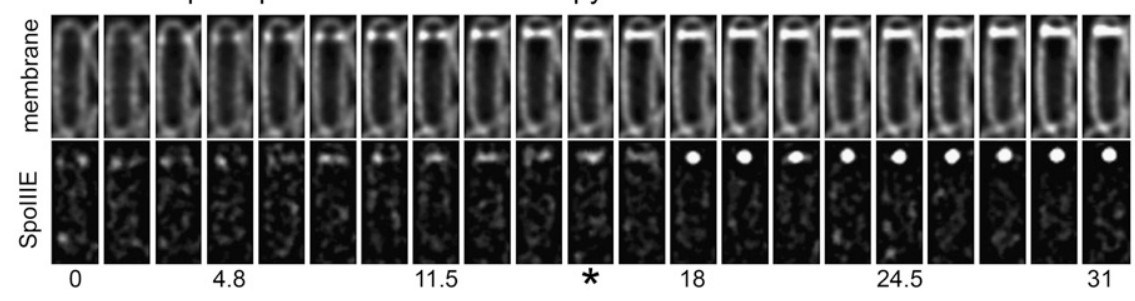

B

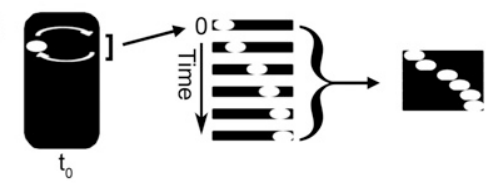

C

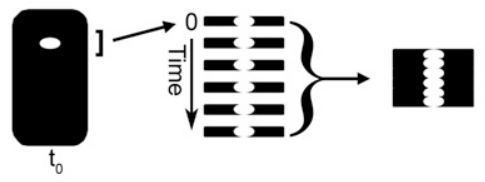

Total internal reflection fluorescence microscopy
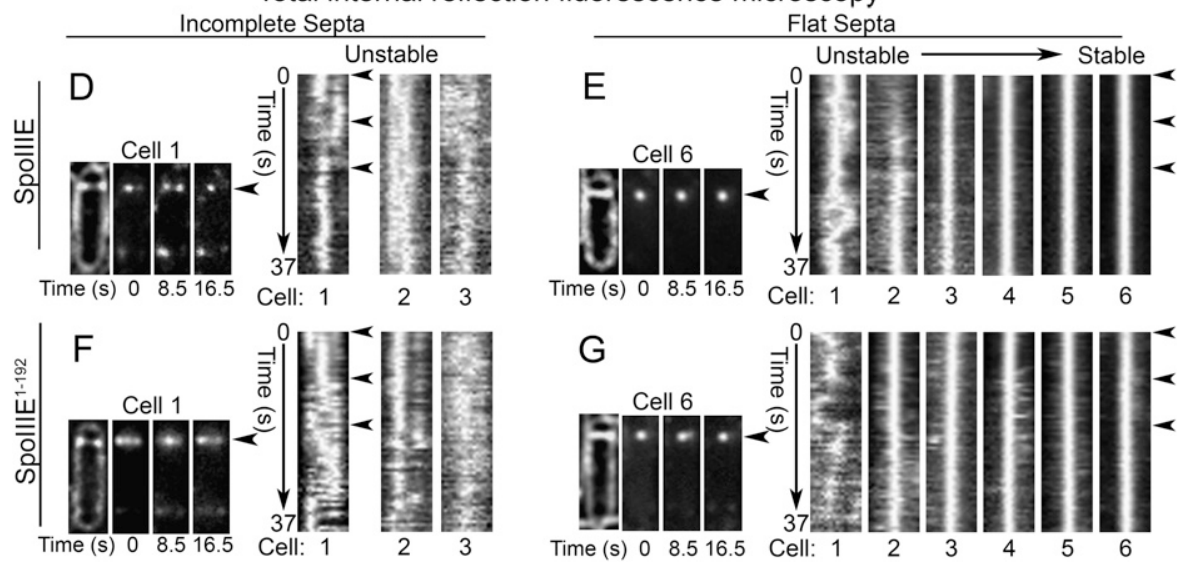

Figure 4. SpoIIIE dynamics. (A) Time-lapse epifluorescence microscopy of SpoIIIE-GFP. Images of FM 4-64 staining (top) and GFP fluorescence (bottom) were collected every $90 \mathrm{sec}$ with time (minutes) shown below the images. The asterick denotes the apparent completion of septation, based on membrane images (see also Supplemental Movie S5). (B,C) Diagrams show construction of septal kymographs in cells with unstable $(B)$ or stable $(C)$ foci. Slices the width of septum were taken for each time point and then stacked, with the first time point at the top and the last at the bottom. $(D-G)$ TIRF microscopy of SpoIIIE-GFP $(D, E)$ and SpoIIIE ${ }^{1-192}$-GFP $(F, G)$ sporangia with clearly incomplete septa $(D, F)$ or flat septa $(E, G)$, based on FM 4-64 staining. Images were collected approximately every $0.5 \mathrm{sec}$ for $>37 \mathrm{sec}$. Septal kymographs for nine individual cells, including three incomplete and six flat septa, were generated by stacking $1 \times 18$ pixel slices of the septum for each time. Arrowheads indicate the times on the kymographs that correspond to the full cell images shown to the left of the kymographs. The first image shows the membrane, and the three following images show TIRF GFP fluorescence at the indicated times (see also Supplemental Movies S6, S7).

SpoIIIE localizes to the leading edge

of constricting septa

To improve the spatial resolution of SpoIIIE imaging experiments, we next performed PALM on cells expressing SpoIIIE, SpoIIIE ${ }^{\mathrm{ATP}-}$, and SpoIIIE ${ }^{1-192}$ fused to tandem dimer EOS (tdEOS) (Wiedenmann et al. 2004). PALM allows localization of individual SpoIIIE-tdEOS molecules with $20-\mathrm{nm}$ precision and the visualization of SpoIIIE localization at higher, non-diffraction-limited spatial resolution (Betzig et al. 2006; Shroff et al. 2008; McKinney et al. 2009). We performed PALM on live cells to avoid membrane perturbations and other artifacts caused by chemical fixation (Supplemental Fig. S5). Live-cell PALM demonstrated that SpoIIIE is clearly localized to constricting and flat septa compared with the rest of the membrane (Fig. 5A-C,L). This high-resolution imaging clearly shows
SpoIIIE molecules to be confined near the leading edge of constricting septa, forming clusters on one or both sides of incomplete septa (Fig. 5, class I), a phenomenon that is much less apparent in diffraction-limited epifluorescence images (Fig. 5, inset). Flat septa displayed two classes of localization. In $52 \%$ of flat septa, two distinct or one elongated cluster of SpoIIIE-tdEOS was distributed along the septal membrane (Fig. 5B, class II). These are likely incomplete septa with an opening smaller than the resolution of the conventional light microscope, since they occur at a frequency similar to septal retraction in wildtype cells in the protoplast assay (Fig. 1B). Another 40\% show a single cluster of SpoIIIE molecules at the septal midpoint, while $8 \%$ display too few molecules to be classified (Fig. 5C, class III). These septa have likely completed septation and assembled a SpoIIIE DNA translocation complex. Thus, live-cell PALM demonstrates 

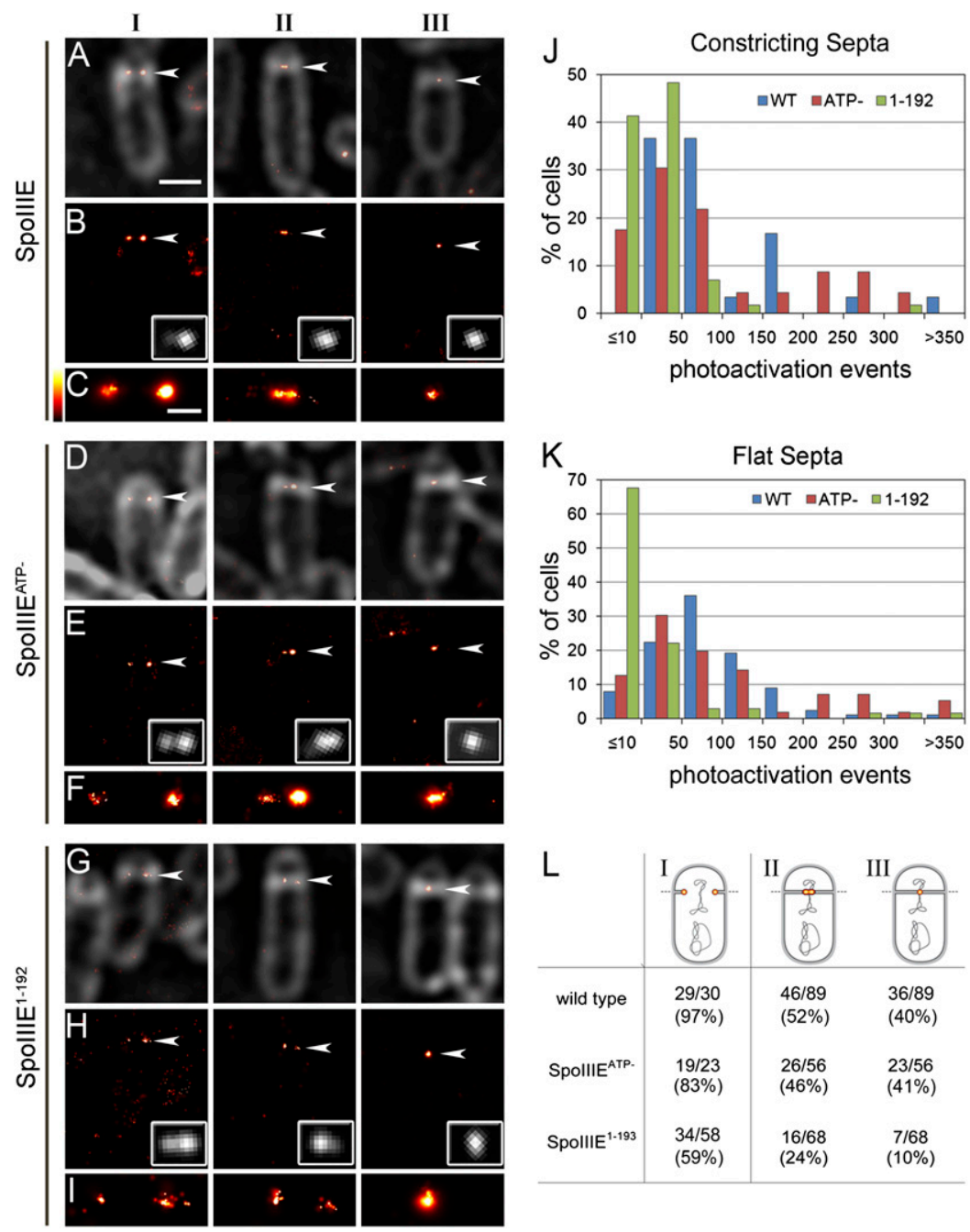
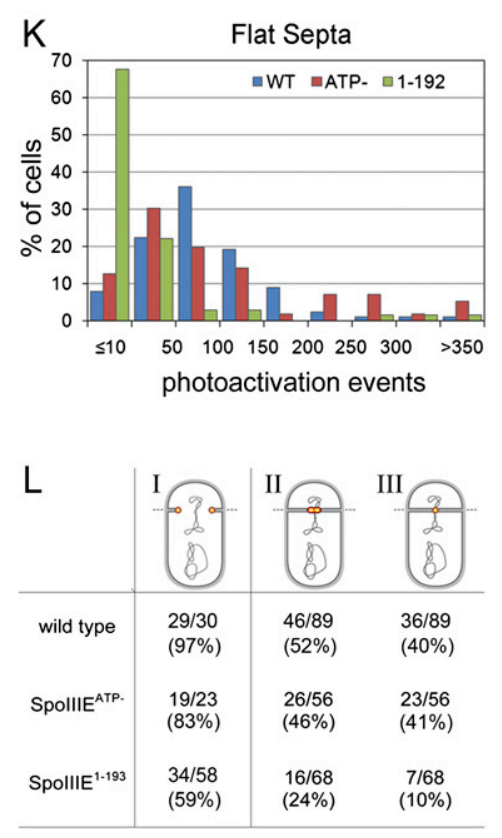

Figure 5. SpoIIIE localization by live-cell PALM. $(A-I)$ Localization of tdEOS fusions to SpoIIIE $(A-C)$, SpoIIIE ${ }^{\text {ATP- }}(D-F)$, and SpoIIIE ${ }^{1-192}$ $(G-I)$ at incomplete (I) and flat (II and III) sporulation septa visualized by FM 5-95 staining. PALM images of SpoIIIE-tdEOS $(B, E, H)$ and diffraction-limited image of membranes (gray) were overlaid $(A, D, G)$ to show the localization of SpoIIIE molecules at the septum (arrowhead). Enlarged PALM images $(C, F, I)$ show individual SpoIIIE-tdEOS molecules not apparent in the diffraction-limited images $(B, E, H$, inset $)$. Bars: $A, 1 \mu \mathrm{m} ; C, 0.2 \mu \mathrm{m} .(J, K)$ The percent of cells with noted range of tdEOS molecules for constricting (class I) and flat septa (class II and III). (L) Number and percent of sporangia with tdEOS fusions proteins at constricting and flat septa. For class 1, a SpoIIIE cluster at one or both sides of an incomplete septum was scored as localized. For cells with flat septa, the presence of a SpoIIIE cluster that spread across the septum or more than one cluster were scored as class II, and those with one compact cluster were scored as class III. Only cells with $>11$ tdEOS molecules were included in classes I-III. tdEOS molecules with localization precision $<50 \mathrm{~nm}$ were selected for analysis. that SpoIIIE first localizes to the leading edge of constricting septa, and then assembles a DNA translocation complex at the septal midpoint.

As expected, SpoIIIE ${ }^{\mathrm{ATP}-}$-tdEOS displayed a localization pattern similar to wild type, indicating that loss of DNA translocation does not affect SpoIIIE assembly (Fig. 5DF,J-L). In contrast to SpoIIIE ${ }^{\mathrm{ATP}-}$-tdEOS and SpoIIIEtdEOS, the SpoIIIE ${ }^{1-192}$-tdEOS fusion showed decreased localization (Fig. 5J-L) and was unable to support septal membrane fission (Supplemental Fig. S5), suggesting that the tdEOS fusion decreases the protein's activity or stability. Consistent with the septal fission defect, SpoIIIE ${ }^{1-192}$. tdEOS formed a single cluster in only $10 \%$ of flat septa by live-cell PALM (Fig. 5L). However, SpoIIIE ${ }^{1-192}$-tdEOS still localized in clusters at the leading edge of $59 \%$ of constricting septa, indicating that the transmembrane domain alone can mediate localization of SpoIIIE to the leading edge of constricting septum (Fig. 5G-L).

\section{Modeling fluorescence recovery in spoIIIE mutants with various cell geometries}

In order to establish a quantitative association between SpoIIIE assembly and membrane fission, we tested whether our FRAP data support a purely diffusive model of membrane dynamics (Lizana et al. 2008), with fluorescence recovery limited by only (1) the time scale required for diffusion across the membrane surface, and (2) the ability of a SpoIIIE complex to block diffusion across the septal pore. To model the dynamics of the density $(\rho)$ of unbleached, membrane-bound FM 4-64 fluorophores, we numerically solve for the steady state of Fick's equation (Murray 2002)

$$
\frac{d \rho(\mathbf{r}, t)}{d t}=D(\mathbf{r}) \nabla^{2} \rho(\mathbf{r}, t)
$$

on a three-dimensional surface defining the cell geometry. $D(\mathbf{r})$ is the diffusion constant, which can vary across the membrane at different positions $\mathbf{r}$ (particularly at the point of septal constriction where SpoIIIE complexes may slow down diffusion), and $\rho=1$ signifies the maximal concentration of lipid labeling with unbleached dye molecules. We simulate FRAP by initializing the membrane with uniform fluorescence $(\rho=1)$, and, after bleaching (at $t=0$ ), the fluorophore density is reduced to 0 across one hemispherical pole. Recovery follows via diffusion of unbleached molecules from the rest of the cell. From the spatiotemporal dynamics of $\rho$, we calculate 
the simulated cFR $c F R^{S}(t)=\rho(x=L, t) / \rho(x=0, t)$, with $x=L$ indicating the forespore pole and $x=0$ indicating the forespore distal pole. Using FRAP simulations on the poles of a vegetative cell geometry, we determined that a diffusion constant $D=0.4 \mu \mathrm{m}^{2} / \mathrm{sec}$ provides a recovery fraction $c F R^{S}(t)$ that closely matches experimental data (Fig. 6A; Supplemental Fig. S6).

Fixing the diffusion constant eliminates the free parameter in Equation 1, and hence the dynamics of invaginated cells are determined entirely by cell geometry and the state of SpoIIIE assembly. Modeling the geometry of a $\Delta$ spoIIIE cell during sporulation as a doublemembrane invagination, FRAP simulations (Fig. 6B) generated a recovery curve strikingly similar to experimental measurements for $\Delta$ spoIIIE cells, with a recovery time constant of $\tau_{1 / 2}=5 \mathrm{sec}$. This result validates our assumption that fluorescence recovery is a diffusionlimited process, with the slowed recovery in $\Delta$ spoIIIE cells relative to vegetative cells caused by the increased distance traversed by nonbleached molecules as they diffuse across the septal bottleneck. In the presence of a wild-type SpoIIIE complex at the midpoint, we assume that diffusion is blocked, and that the limited recoveries observed for wild-type sporangia (Fig. 1G,H) are not a result of diffusion. Instead, the recovery values are likely due to either incomplete coverage of the pole region with the laser spot or incomplete bleaching of the FM 4-64 molecules.
We next tested whether the wide variability in recovery rates of spoIIIE ${ }^{1-192}$, spoIIIE ${ }^{\mathrm{Fis}-}$, and spoIIIE ${ }^{\mathrm{Fis}-\mathrm{ATP}-}$ mutants results from assembly of abnormal "leaky" channels that are differentially permeable to FM 4-64. We therefore modeled the effects of reducing the FM 4-64 diffusion coefficient at the septal midpoint. Our results indicated that the diffusion coefficient had to be reduced by four orders of magnitude before intermediate recovery curves were observed. However, the simulated curves did not quantitatively match the experimental data (Fig. 6C), displaying more sigmoidal character.

Instead of a static leaky complex, the spatial movements of SpoIIIE observed in TIRF experiments suggested that diffusion across the septum may be dynamically controlled by the assembly state of the SpoIIIE complex. To test this hypothesis, we performed FRAP simulations in which mutant SpoIIIE proteins could be fully assembled and blocking diffusion, or completely disassembled and allowing rapid diffusion. The probability $(p)$ of finding SpoIIIE assembled in any time step of $0.2 \mathrm{sec}$, matching the acquisition time of TIRF experiments, was varied from $p=1$ (always assembled, as in wild-type SpoIIIE) to $p$ $=0$ (always disassembled, as in $\Delta$ spoIIIE). Interestingly, FRAP simulations in which $p$ was varied from 0.5 to 1.0 in 0.05 increments produced a range of recovery curves (Fig. 6D) that closely mimic those observed in SpoIIIE ${ }^{1-192}$ and SpoIIIE ${ }^{\text {Fis- }}$ cells (Fig. 3A-C). In agreement with these results, we estimated that the probability of assembly for
A
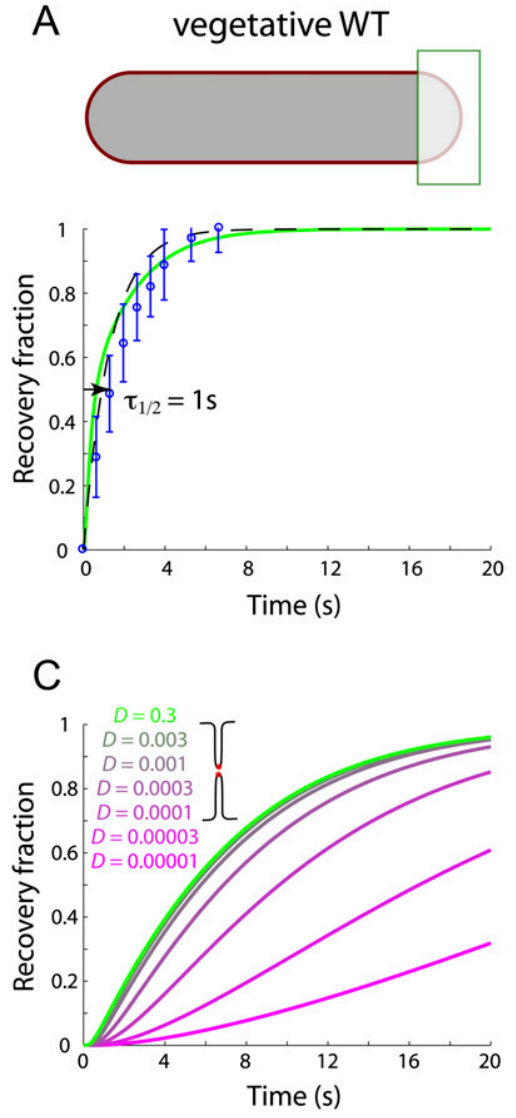
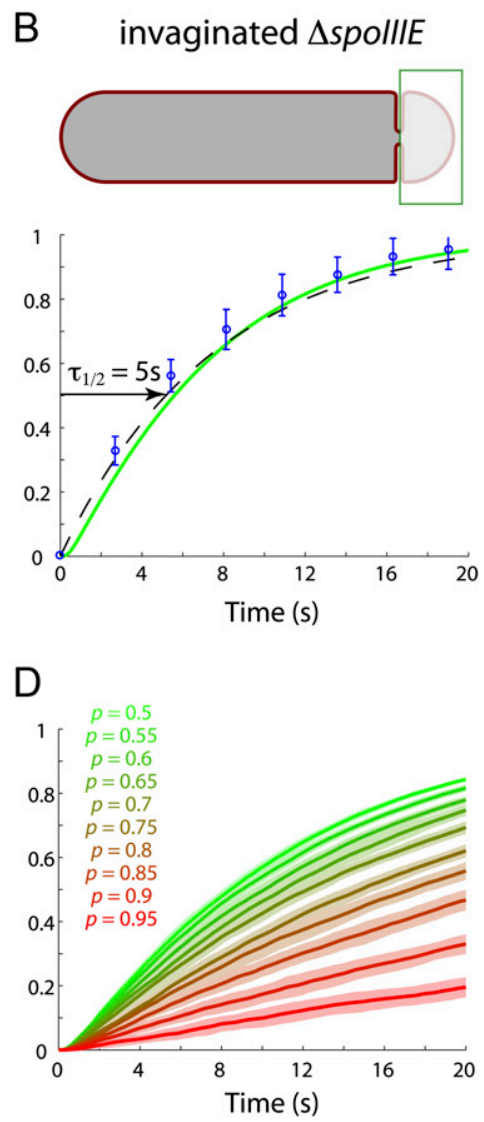

Figure 6. Modeling FM 4-64 diffusion in various cell geometries. (A) Modeling of FRAP on the poles of cells $2.8 \mu \mathrm{m}$ long by $0.854 \mu \mathrm{m}$ wide, represented by a cylindrical surface with hemispherical poles. Simulated recovery curves (green) follow the functional form $\left(1-2^{-\tau / \tau_{1 / 2}}\right)$. (A) The simulated $\mathrm{cFR}$ $\left(c F R^{S}\right.$, green) of lipid molecules with a diffusion constant $D=0.4 \mu \mathrm{m}^{2} / \mathrm{sec}$ in a vegative cell geometry closely matches experimental data (blue circles) and has a recovery time constant of $\tau_{1 / 2}=1 \mathrm{sec}$ (exponential fit shown as dashed line). $(B-D)$ FRAP simulations of an invaginated cell with a pore radius of $10 \mathrm{~nm}$ (see Supplemental Fig. S6 for other pore radii). (B) $c F R^{S}$ of lipids with $D=0.4 \mu \mathrm{m}^{2} / \mathrm{sec}$ predicts a recovery time constant of $\tau_{1 / 2}=5 \mathrm{sec}$ that also closely matches $\Delta$ spoIIIE experimental data (blue circles). $(C) C F R^{S}$ assuming a leaky complex with a range of diffusion constants, $D$, at the tip of the septal invagination. $(D) c F R^{S}$ with different probabilities $(p)$ of SpoIIIE assembly and consequent inhibition of diffusion. 
the cells represented by the kymographs in Figure $4 \mathrm{G}$ is $0.22,0.55,0.78,0.88,0.96$, and 0.98 (for cells $1-6$, respectively). Taken together, these simulations support a model in which the intermediate FRAP curves observed in spoIIIE ${ }^{1-192}$, spoIIIE ${ }^{\mathrm{Fis}-}$, and spoIIIE ${ }^{\mathrm{Fis}-\mathrm{ATP}-}$ strains reflect repeated disassembly and assembly of a SpoIIIE channel at the septal midpoint, rather than the static assembly of a channel that is only partially permeable to lipid molecules.

\section{Discussion}

Our data indicate that SpoIIIE assembles a DNA translocation complex via a multistep pathway that requires both the transmembrane domain, which mediates dynamic localization to division sites and the assembly of unstable foci, and the cytoplasmic domain, which stabilizes the DNA translocation complex. We further demonstrated that the absence of SpoIIIE causes a severe defect in septal membrane fission when DNA traverses the septum, that daughter cell membranes are separated during DNA translocation, and that there is a quantitative association between septal membrane fission and the assembly of a stable DNA translocation complex. These findings provide the first direct experimental evidence that SpoIIIE assembly mediates septal membrane fission during sporulation (Liu et al. 2006; Burton et al. 2007).

The first step in the SpoIIIE assembly pathway is dynamic localization to active division sites, a process mediated by the transmembrane domain (Fig. 7A-C, step 1). Epifluorescence microscopy initially suggested that SpoIIIE localizes as a ring at constricting septa (Sharp and Pogliano 2002). However, the improved temporal resolution of TIRF microscopy reveals that these "rings" represent the blurred motion of rapidly moving molecules imaged with exposure times that are long relative to the rate of movement. The improved spatial resolution of live-cell PALM indicates that SpoIIIE localizes specifically to the leading edge of constricting septa. It is tempting to speculate that this localization might be mediated by the ability of the transmembrane domain to sense regions of increased membrane curvature, as demonstrated for several membrane proteins and wedge-shaped lipids (Huang et al. 2006; Ramamurthi et al. 2009).

The second step in SpoIIIE assembly is the formation of a focus at the septal midpoint, a process mediated by the transmembrane domain. Live-cell PALM demonstrates that there are similar numbers of SpoIIIE molecules moving at constricting septa as in the focus, suggesting that most SpoIIIE molecules at the nascent septum ultimately assemble into the septal focus. In keeping with previous studies (Bath et al. 2000; Sharp and Pogliano 2003; Liu et al. 2006), we find that the transmembrane domain is capable of assembling foci at the septal midpoint, although TIRF microscopy demonstrates that these foci are less stable than those assembled by the full-length protein. Our data therefore suggest a third step in SpoIIIE assembly: stabilization of the transmembrane assembly by the cytoplasmic domain. We suggest that this step involves the engagement of the DNA motor domain with

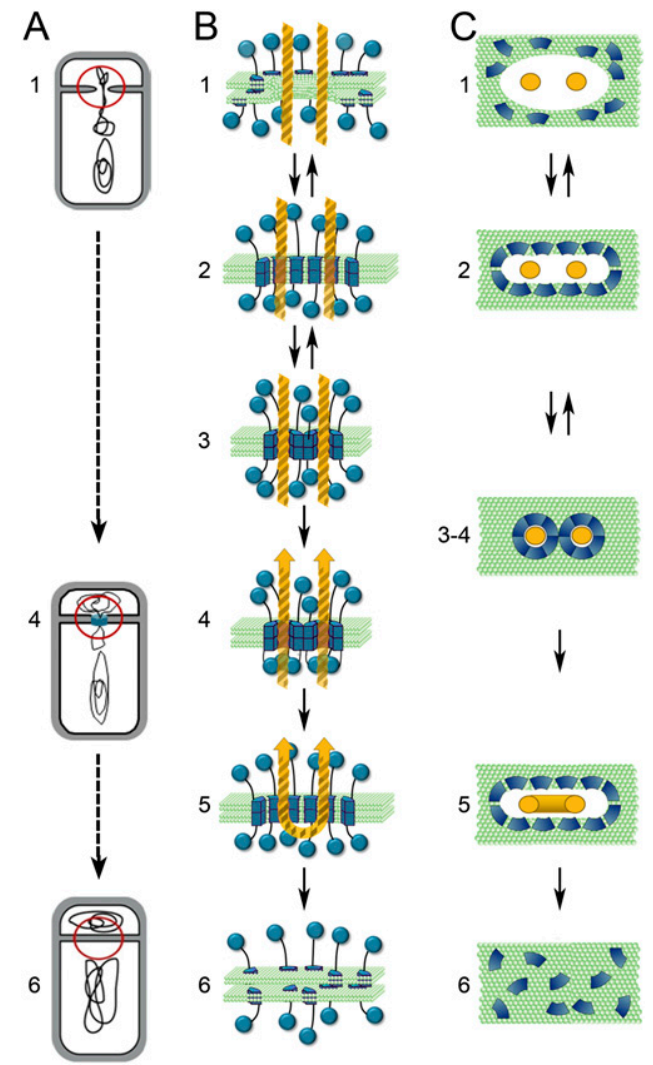

Figure 7. Model for SpoIIIE function in septal membrane fission. Diagrams of septation show the whole cell $(A)$, with the region of interest indicated by red circles, and the septum as a side view $(B)$ and as a top view $(C)$, showing the lipid bilayer (green circles), DNA (gold), and SpoIIIE (blue). SpoIIIE transmembrane domain is shown as a wedge in the lipid bilayer, with the linker as a black line and the motor domain as a sphere. (Step 1) Initially, SpoIIIE dynamically localizes to the leading edge of constricting septa, but does not block lipid diffusion. As the membrane contacts DNA, the SpoIIIE transmembrane domain assembles around the trapped DNA (step 2) forming an unstable channel that can separate membranes (step 3). (Step 4) The cytoplasmic motor domain then assembles on the DNA (likely only in the mother cell) (Becker and Pogliano 2007), stabilizing the channel and forming a channel that blocks diffusion between the daughter cell membranes during DNA translocation. We show this structure to be a paired channel, although other arrangements are possible. (Step 5) After DNA translocation, the motor domain releases DNA, and the complex disassociates to allow the terminus across the septum. (Step 6) Finally, irreversible membrane fission occurs.

DNA that traverses the septum, which stabilizes the SpoIIIE complex for the duration of DNA translocation (Fig. 7A-C, steps 3, 4). This stabilization might also require the linker domain, which appears to stabilize the motor domain of Escherichia coli FtsK (Dubarry and Barre 2010). This DNA-dependent stabilization of the SpoIIIE focus likely explains previous observations that SpoIIIE assembles only in the presence of trapped DNA (BenYehuda et al. 2003a), since transient foci and dynamic SpoIIIE molecules at nascent septa are difficult to image. 
SpoIIIE therefore has at least two domains that cooperate to mediate the assembly of the DNA translocation complex: the transmembrane domain that targets the protein to the septum (and likely assembles a channel at the septal midpoint), and the DNA motor domain that can hexamerize in the presence of DNA even in the absence of the transmembrane domain (Ptacin et al. 2008). A critical question for future studies is how the assembly and disassembly of these domains are coordinated and coupled to the initiation and termination of DNA translocation.

Our membrane FRAP experiments and biophysical modeling results indicate that FM 4-64 diffuses through the cytoplasmic membrane with a diffusion coefficient of $0.4 \mathrm{\mu m}^{2} / \mathrm{sec}$, a rate that suggests that the dye molecules do not significantly impair free diffusion within the cytoplasmic membrane due to interactions with localized proteins or lipids. They also demonstrate that, in the absence of SpoIIIE, septal membrane fission does not occur, allowing FM 4-64 to diffuse into the bleached forespore membrane at rates consistent with the existence of a $10-\mathrm{nm}$ opening in the septum. These results contradict a previous study in which fewer cells were bleached and fewer spoIIIE mutants were examined (see Supplemental Fig. S1 for discussion; Burton et al. 2007). However, consistent with this prior study, we find that translocation-defective and assembly-proficient SpoIIIE proteins are capable of blocking lipid diffusion through the septum. This suggests that the DNA translocation complex moves the DNA across separated daughter cell membranes. This finding is consistent with the paired channel model for SpoIIIE topology (Liu et al. 2006; Burton et al. 2007), which postulates that SpoIIIE forms a channel that spans two lipid bilayers (Fig. 7). Recent experiments indicate that the transmembrane domain of the SpoIIIE homolog FtsK can be replaced with other transmembrane domains (Dubarry and Barre 2010). This suggests that DNA translocation does not depend on the assembly of a channel, which is consistent with our observations that the membrane fission-defective SpoIIIE mutant is DNA translocation-proficient (Liu et al. 2006).

Our localization studies, FRAP analysis, and biophysical modeling efforts provide quantitative support for the model that assembly of SpoIIIE into a focus at the septal midpoint mediates septal membrane fission (Liu et al. 2006). Most compelling of these findings is that the SpoIIIE transmembrane domain, which TIRF microscopy shows to assemble unstable foci, produces a wide range of FRAP recovery curves. Biophysical modeling of FRAP data indicates that this outcome can be readily recapitulated in simulations in which the SpoIIIE transmembrane domain alternates between fully assembled and disassembled states that either completely block or allow FM 4-64 diffusion across the septum, respectively (Fig. 7A-C, steps 2,3$)$. This suggests that the variable FRAP outcomes in different cells reflect subtle changes in the probability of assembly of the transmembrane domain in each cell, perhaps due to cell-cell variations in the number of SpoIIIE molecules at the septum observed by PALM (Fig. 5). If SpoIIIE does assemble a channel for the DNA, then the assembly and disassembly we observe would require that the hydrophilic amino acids that line the aqueous pore be masked in the disassembled subunits to prevent the energetically unfavorable interaction of these amino acids with the hydrophobic core of the lipid bilayer. This likely requires either the presence of membrane proteins that act as chaperones for the unfolded subunits, or rearrangements of the individual transmembrane segments to bury these regions.

Several genetic, biophysical, and cell biological experiments indicate that SpoIIIE translocates the two arms of the circular chromosome simultaneously (Wu and Errington 1998; Becker and Pogliano 2007; Burton et al. 2007; Ptacin et al. 2008), although structural studies demonstrate that the DNA motor domain can only accommodate a single dsDNA strand (Massey et al. 2006). It is therefore likely that the septum contains at least two SpoIIIE channels, one for each arm of the circular chromosome that traverses the septum. Translocating a circular chromosome through two adjacent channels poses a difficulty when the final loop of DNA reaches the septum. Our observation that the absence of the DNAbinding domain produces a channel that can assemble and disassemble suggests that, once the terminus reaches the septum, the DNA motor domain disassociates from the DNA and destabilizes the channel, opening a pore that allows the terminus to pass into the forespore (Fig. 7A-C, step 5). After DNA is cleared from the septum, SpoIIIE or another protein involved in septal membrane fission in the absence of DNA could permanently separate the membranes (Fig. 7A-C, step 6).

Finally, our data emphasize the importance of thoughtfully considering the caveats of various imaging methods before interpreting cell biological data. First, although both epifluorescence microscopy and live-cell PALM appear to show that SpoIIIE localizes as rings at nascent division sites, TIRF microscopy shows that these rings are artifacts caused by blurring of dynamic molecules during long exposure times in epifluorescence microscopy and the compilation of molecules imaged over several minutes into a single image in PALM. Second, although the dynamic assemblies at constricting septa appear less intense than foci in both epifluorescence and TIRF microscopy, live-cell PALM demonstrates that there are similar numbers of SpoIIIE molecules at both assembly stages. Live-cell PALM has two attributes that likely account for this difference: the ability to detect and enumerate single fluorescent molecules, which eliminates the preferential detection of stationary rather than dynamic molecules, and the more effective elimination of noise during imaging processing that is facilitated by the intense burst of photons emitted by tdEOS during the short (50-msec) exposure time. Thus, PALM provides both improved spatial resolution and a more quantitative measure of SpoIIIE abundance, TIRF probes SpoIIIE dynamics at higher temporal resolution to visualize rapid steps in complex assembly, and epifluorescence provides a broad perspective of SpoIIIE dynamics throughout cell division and engulfment. Each method reveals different features of the SpoIIIE assembly pathway, which is 
characterized by a wide range of movement rates, from the very rapid movement around nascent septa (within seconds) to the more leisurely movement of the focus to the cell pole during engulfment (within $45 \mathrm{~min}$ ). Integration of these approaches with biophysical modeling provides a mechanistic explanation of membrane fission that bridges the molecular and cellular scales.

\section{Materials and methods}

\section{Strains and culture conditions}

Strains are derivatives of B. subtilis PY79 (Supplemental Table S2). Mutations were introduced by transformation with plasmids or genomic DNA (Dubnau and Davidoff-Abelson 1971). See the Supplemental Material for details of plasmid construction, including oligonucleotide sequences (Supplemental Table S3). Cells were grown in 20\%-25\% LB, and sporulation was induced by resuspension (Becker and Pogliano 2007) at $37^{\circ} \mathrm{C}$ unless noted otherwise.

\section{Microscopy}

Except for PALM data, microscopy was performed on an Applied Precision optical sectioning microscope (Liu et al. 2006) using softWoRx version 3.3.6 (Applied Precision) and ImageJ for analysis. Cells were sporulated in the presence of $0.5 \mu \mathrm{g} / \mathrm{mL}$ membrane dye FM 4-64 and 0.5 $\mu$ M nucleic acid stain Sytox Green (Invitrogen), as needed. Samples were immobilized on poly-L-lysine-treated coverslips, except for TIRF and time-lapse microscopy, which were performed on agarose pads (Becker and Pogliano 2007). For time lapse, cells were placed on the agarose pad 1.5-2 $\mathrm{h}$ after resuspension and were incubated at $30^{\circ} \mathrm{C}$ during imaging. Images were collected every $90 \mathrm{sec}$ for $>30 \mathrm{~min}$. TIRF microscopy used an Olympus $100 \times 1.65$ Apo objective, immersion oil $n=1.78$ (Cargille Laboratories), and sapphire coverslips (Olympus). TIRF images were collected approximately every 500 msec, until the sample photobleached using a 488-nm argon laser attached to an Applied Precision TIRF module set at $70 \%$ power for pulses of $0.2 \mathrm{sec}$. Kymographs were generated with Image J, making a montage of $0.06-\mu \mathrm{m} \times 1.14-\mu \mathrm{m}$ slices through the septum at each time.

\section{Protoplast assay}

Two hours to $2.5 \mathrm{~h}$ after initiation of sporulation, cells were concentrated $20 \times$ in SMM buffer, placed on a slide, and mixed with lysozyme (final concentration, $1 \mathrm{mg} / \mathrm{mL}$ ) (Broder and Pogliano 2006). Images were taken every $60 \mathrm{sec}$ until all cells formed protoplasts, which was variable. Sporangia were scored as having either complete septal fission (if the septum remained throughout protoplast formation to generate two protoplasts) or incomplete septal fission (by septal retraction).

\section{Quantification of FRAP}

To maintain sufficient fluorescence to measure recovery after photobleaching, we sporulated cells in $2 \mu \mathrm{g} / \mathrm{mL}$ FM 4-64. After $2-2.5 \mathrm{~h}$ of sporulation at $37^{\circ} \mathrm{C}$ or $3 \mathrm{~h}$ of sporulation at $30^{\circ} \mathrm{C}$, cells were pelleted by microcentrifugation at $7000 \mathrm{rpm}$ for $10 \mathrm{sec}$, washed twice in sporulation media, resuspended in 1/20th vol of fresh sporulation media, and mounted on a poly-Lysine coverslip. Photobleaching was achieved with one 0.3 -sec pulse from a 488 -nm argon laser set to $20 \%-30 \%$ power. FRAP quantification was performed as described by Broder and Pogliano (2006) with the following modifications. To compare multiple FRAP events on a single graph, we calculated the cFR by determining the relative intensity of the bleached region to the unbleached region, and defining the bleach event as $\mathrm{cFR}=0$ and complete recovery as $\mathrm{cFR}=1$ for each photobleached sporangia (Supplemental Material; Wu et al. 2006). Average cFR curves were obtained by averaging fluorescence recovery values at the same time points for each strain. The equation $c F R(t)=A\left(1-e^{-t / t 1}\right)\left(\mathrm{y}_{0}\right.$ $\left.+B e^{-t / t 2}\right)$ was used for curve fitting.

\section{Quantification of GFP fluorescence intensity}

To avoid bleed-through of FM 4-64 fluorescence in the FITC channel, we sporulated cells in $0.25 \mu \mathrm{g} / \mathrm{mL}$ FM4-64. Images of the medial focal plane were taken $2 \mathrm{~h}$ after resuspension with a 3 -sec exposure for GFP. GFP intensities were first modified by $3 \times 3$ binning using ImageJ to smooth fluorescence intensity when the peak is spread over 2 pixels versus centered over 1 pixel. GFP foci were identified using the ImageJ threshold tool. Only foci from sporangia with flat or slightly curved septa were quantified. The maximum fluorescence values for at least 200 foci for each strain were corrected for background fluorescence, normalized to the photosensor reading for each image to account for any changes in bulb intensity, and then averaged.

\section{PALM acquisition and analysis}

The PALM instrument was built on a commercial Olympus IX71 fluorescence microscope with two lasers, wavelengths $561 \mathrm{~nm}$ (Compass 561, $20 \mathrm{~mW}$, Coherent, Inc.) and $405 \mathrm{~nm}$ (Cube, 405 $\mathrm{nm}, 100 \mathrm{~mW}$, Coherent, Inc.), and an Olympus UAPO150xO/ TIRFM-SP NA1.45 objective. Fluorescence images were acquired by a low-noise, light-sensitive EMCCD (DV887ECS-BV, Andor). Cells were sporulated by resuspension at $30^{\circ} \mathrm{C}$, and the membranes were stained with $0.05 \mu \mathrm{g} / \mathrm{mL}$ FM 5-95 and harvested at $3 \mathrm{~h}$ after sporulation. After two washes and then concentration, cells mixed with gold nanospheres (790122, MicrospheresNanospheres) were immobilized on poly-L-lysine-treated coverslips. FM 5-95-labeled membranes were imaged with the fluorescence filter set (FF01-560/25-25 and FF01-684/25-25, Semrock), and then photobleached by illumination with intense 561-nm laser $(\sim 4.2 \mathrm{~mW})$ for a few minutes prior to the PALM data acquisition to eliminate background fluorescence. After focusing to achieve partial TIRF, the 405-nm activation laser was turned on low $(1.2-6.0 \mu \mathrm{W})$ and PALM data were acquired using an emission filter for tdEOS (FF01-588/21-25, Semrock). About 10,000 frames at $50 \mathrm{msec}$ per frame were acquired and analyzed with programs written in MATLAB based on previously described algorithms (Betzig et al. 2006). The molecular positions of local peaks (approximately eight times brighter than the background noise) were determined by fitting to two-dimensional Gaussian PSF, and in-plane sample drift was corrected by the localization of the bright Au-fiducials at nanometer accuracy (Yildiz et al. 2003). See the Supplemental Material for further details.

\section{Acknowledgments}

We thank Jan Liphardt, Ann McEvoy, and Derek Greenfield for discussion and assistance with the PALM instrumentation, and Michael W. Davidson for the gift of the tdEOS plasmid. We also thank Dan Broder for help with the protoplast experiments. T.F. and E.B. performed the epifluorescence, FRAP, and TIRF experiments in the K.P. laboratory. K.C.H. performed the biophysical modeling experiments. J.Y.S and S.H.L. conducted PALM experiments in the C.B. laboratory. This research was supported by National Institutes of Health grants GM057045 (to K.P.), 
GM071552 and GM032543 (to C.B.), and GM075000 (to K.C.H), and the Director's New Innovator Award DP2OD006466 (to K.C.H). T.C.F. is supported by the NIH post-doctoral fellowship 5F32GM081174.

\section{References}

Bath J, Wu LJ, Errington J, Wang JC. 2000. Role of Bacillus subtilis SpoIIIE in DNA transport across the mother cellprespore division septum. Science 290: 995-997.

Becker EC, Pogliano K. 2007. Cell-specific SpoIIIE assembly and DNA translocation polarity are dictated by chromosome orientation. Mol Microbiol 66: 1066-1079.

Ben-Yehuda S, Rudner DZ, Losick R. 2003a. Assembly of the SpoIIIE DNA translocase depends on chromosome trapping in Bacillus subtilis. Curr Biol 13: 2196-2200.

Ben-Yehuda S, Rudner DZ, Losick R. 2003b. RacA, a bacterial protein that anchors chromosomes to the cell poles. Science 299: 532-536.

Betzig E, Patterson GH, Sougrat R, Lindwasser OW, Olenych S, Bonifacino JS, Davidson MW, Lippincott-Schwartz J, Hess HF. 2006. Imaging intracellular fluorescent proteins at nanometer resolution. Science 313: 1642-1645.

Broder DH, Pogliano K. 2006. Forespore engulfment mediated by a ratchet-like mechanism. Cell 126: 917-928.

Burton BM, Marquis KA, Sullivan NL, Rapoport TA, Rudner DZ. 2007. The ATPase SpoIIIE transports DNA across fused septal membranes during sporulation in Bacillus subtilis. Cell 131: 1301-1312.

Dubarry N, Barre FX. 2010. Fully efficient chromosome dimer resolution in Escherichia coli cells lacking the integral membrane domain of FtsK. EMBO J 29: 597-605.

Dubnau D, Davidoff-Abelson R. 1971. Fate of transforming DNA following uptake by competent Bacillus subtilis. I. Formation and properties of the donor-recipient complex. I Mol Biol 56: 209-221.

Errington J. 2001. Septation and chromosome segregation during sporulation in Bacillus subtilis. Curr Opin Microbiol 4: 660666.

Errington J. 2003. Regulation of endospore formation in Bacillus subtilis. Nat Rev Microbiol 1: 117-126.

Finger FP, White JG. 2002. Fusion and fission: Membrane trafficking in animal cytokinesis. Cell 108: 727-730.

Hilbert DW, Piggot PJ. 2004. Compartmentalization of gene expression during Bacillus subtilis spore formation. Microbiol Mol Biol Rev 68: 234-262.

Huang KC, Mukhopadhyay R, Wingreen NS. 2006. A curvaturemediated mechanism for localization of lipids to bacterial poles. PLoS Comput Biol 2: e151. doi: 10.1371/journal.pcbi. 0020151.

Jurgens G. 2005. Plant cytokinesis: Fission by fusion. Trends Cell Biol 15: 277-283.

Liu NJ, Dutton RJ, Pogliano K. 2006. Evidence that the SpoIIIE DNA translocase participates in membrane fusion during cytokinesis and engulfment. Mol Microbiol 59: 1097-1113.

Lizana L, Bauer B, Orwar O. 2008. Controlling the rates of biochemical reactions and signaling networks by shape and volume changes. Proc Natl Acad Sci 105: 4099-4104.

Massey TH, Mercogliano CP, Yates J, Sherratt DJ, Lowe J. 2006. Double-stranded DNA translocation: Structure and mechanism of hexameric FtsK. Mol Cell 23: 457-469.

McKinney SA, Murphy CS, Hazelwood KL, Davidson MW, Looger LL. 2009. A bright and photostable photoconvertible fluorescent protein. Nat Methods 6: 131-133.

Murray JD. 2002. Mathematical biology. Springer, New York.
Pogliano K, Hofmeister AE, Losick R. 1997. Disappearance of the $\sigma^{\mathrm{E}}$ transcription factor from the forespore and the SpoIIE phosphatase from the mother cell contributes to establishment of cell-specific gene expression during sporulation in Bacillus subtilis. J Bacteriol 179: 3331-3341.

Ptacin JL, Nollmann M, Becker EC, Cozzarelli NR, Pogliano K, Bustamante C. 2008. Sequence-directed DNA export guides chromosome translocation during sporulation in Bacillus subtilis. Nat Struct Mol Biol 15: 485-493.

Ramamurthi KS, Lecuyer S, Stone HA, Losick R. 2009. Geometric cue for protein localization in a bacterium. Science 323: 1354-1357.

Sharp MD, Pogliano K. 1999. An in vivo membrane fusion assay implicates SpoIIIE in the final stages of engulfment during Bacillus subtilis sporulation. Proc Natl Acad Sci 96: 1455314558.

Sharp MD, Pogliano K. 2002. Role of cell-specific SpoIIIE assembly in polarity of DNA transfer. Science 295: 137-139.

Sharp MD, Pogliano K. 2003. The membrane domain of SpoIIIE is required for membrane fusion during Bacillus subtilis sporulation. J Bacteriol 185: 2005-2008.

Shroff H, White H, Betzig E. 2008. Photoactivated localization microscopy (PALM) of adhesion complexes. Curr Protoc Cell Biol 41: 4.21.1-4.21.27. doi: 10.1002/0471143030.cb0421s41.

Wiedenmann J, Ivanchenko S, Oswald F, Schmitt F, Rocker C, Salih A, Spindler KD, Nienhaus GU. 2004. EosFP, a fluorescent marker protein with UV-inducible green-to-red fluorescence conversion. Proc Natl Acad Sci 101: 15905-15910.

Wu LJ, Errington J. 1994. Bacillus subtilis SpoIIIE protein required for DNA segregation during asymmetric cell division. Science 264: 572-575.

Wu LJ, Errington J. 1997. Septal localization of the SpoIIIE chromosome partitioning protein in Bacillus subtilis. EMBO J 16: 2161-2169.

Wu LJ, Errington J. 1998. Use of asymmetric cell division and spoIIIE mutants to probe chromosome orientation and organization in Bacillus subtilis. Mol Microbiol 27: 777-786.

Wu LJ, Errington J. 2003. RacA and the Soj-Spo0J system combine to effect polar chromosome segregation in sporulating Bacillus subtilis. Mol Microbiol 49: 1463-1475.

Wu YX, Masison DC, Eisenberg E, Greene LE. 2006. Application of photobleaching for measuring diffusion of prion proteins in cytosol of yeast cells. Methods 39: 43-49.

Yildiz A, Forkey JN, McKinney SA, Ha T, Goldman YE, Selvin PR. 2003. Myosin V walks hand-over-hand: Single fluorophore imaging with 1.5-nm localization. Science 300: 20612065. 


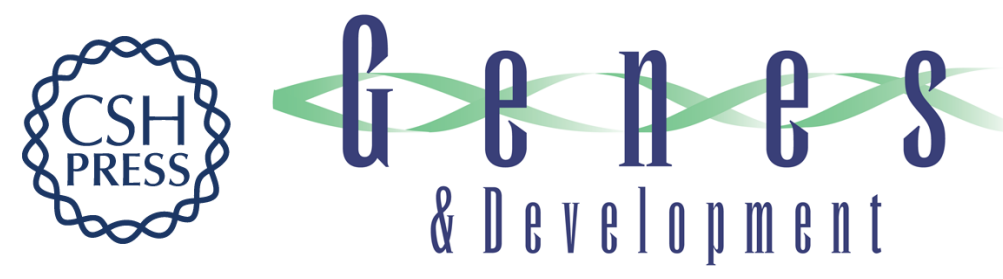

\section{Dynamic SpollIE assembly mediates septal membrane fission during Bacillus subtilis sporulation}

Tinya C. Fleming, Jae Yen Shin, Sang-Hyuk Lee, et al.

Genes Dev. 2010, 24:

Access the most recent version at doi:10.1101/gad.1925210

Supplemental http://genesdev.cshlp.org/content/suppl/2010/05/26/24.11.1160.DC1
Material

References This article cites 33 articles, 14 of which can be accessed free at:

http://genesdev.cshlp.org/content/24/11/1160.full.html\#ref-list-1

License

Email Alerting

Receive free email alerts when new articles cite this article - sign up in the box at the top

Service

right corner of the article or click here.

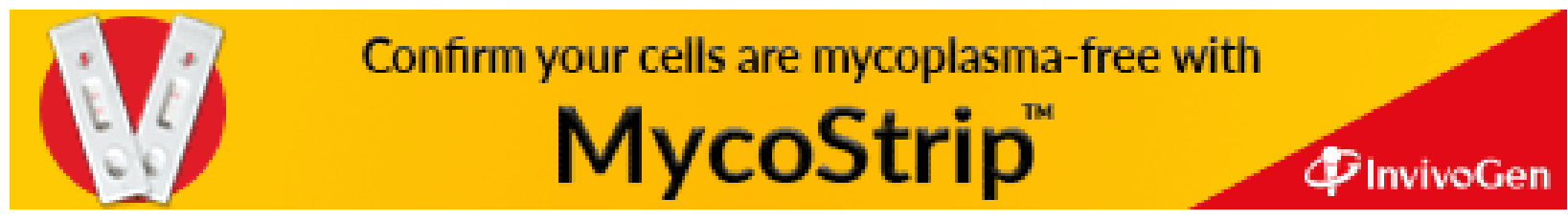

